\section{Tumor odontogénico adenomatoide. Reporte de caso}

\section{Adenomatoid odontogenic tumor. Case report}

\section{Resumen}

El tumor odontogénico adenomatoide (TOA), es un tumor odontogénico epitelial benigno que se presenta en pacientes jóvenes de sexo femenino y con mayor incidencia en la región maxilar. El TOA es de crecimiento lento, progresivo, poco invasivo y asintomático que se relaciona con órganos dentarios no erupcionados. El tratamiento de elección es la enucleación y curetaje de la zona afectada con una recurrencia rara. Este reporte de caso describe a una paciente de 8 años con un TOA ubicado en la región maxilar del lado derecho; se describe la sintomatología, la evaluación imagenológica y el tratamiento quirúrgico propuesto. La evolución fue favorable; no se presentó recidiva del tumor.

Palabras clave: Diente impactado; Neoplasias de la boca; Neoplasias maxilares.

\begin{abstract}
The adenomatoid odontogenic tumor (AOT) is a benign epithelial odontogenic tumor that occurs in young female patients with a higher incidence in the maxillary region. The AOT is slow-growing, progressive, minimally invasive and asymptomatic tumor, that is related to un erupted tooth organs. The choice treatment is enucleation and curettage of the affected area with a rare recurrence. This case report describes an 8-year-old patient with a AOT located in the maxillary region on the right side; the symptomatology, the imaging evaluation and the proposed surgical treatment are described. The evolution was favorable; there was no tumor recurrence.
\end{abstract}

Keywords: Maxillary neoplasms; Mouth neoplasms; Impacted tooth.

\section{Caso Clínico}

\author{
Jonathan Deza Palle ${ }^{1, a}$, Marco Arce Lazo ${ }^{2, b}$ \\ ${ }^{1}$ Facultad de Odontología. Universidad Nacional \\ Mayor de San Marcos. Lima, Perú. \\ ${ }^{2}$ Facultad de Odontología. Universidad Católica \\ de Santa María. Arequipa, Perú \\ a Residente de Cirugía Bucal y Maxilofacial. \\ ${ }^{\mathrm{b}}$ Cirujano Maxilofacial

\section{Correspondencia:} \\ Jonathan Deza Palle \\ Correo electrónico: jona_17_02@hotmail.com \\ Jr. Tomas Ramsey 905, Dpto. 1404. Magdale- \\ na del Mar, Lima, Perú.
}

\section{Coautor:}

Marco Arce Lazo

asdent_7@hotmail.com

Conflicto de intereses: El autor declara no tener conflicto de interés.

Fuente de financiamiento: Autofinanciado

Fecha de recepción: 17/09/17

Fecha de aceptación: 31/10/17 


\section{Introducción}

El tumor odontogénico adenomatoide (TOA) es un tumor odontogénico epitelial benigno, asintomático, poco frecuente, que representa del 2 al $7 \%$ de todos los tumores benignos odontogénicos ${ }^{1}$. En la clasificación de la OMS, se define como un tumor compuesto de epitelio odontogénico con una variedad de patrones histológicos dentro de un estroma de tejido conectivo maduro ${ }^{2}$.

El TOA se presenta con mayor incidencia en el sexo femenino durante la segunda y tercera década de vida. El maxilar superior es el sitio más afectado, casi el doble de veces que la mandíbula; así mismo la región anterior es la zona más afectada con respecto a la zona posterior 3. El tumor se caracteriza por ser de crecimiento lento, pero su comportamiento es agresivo, lo que provoca la expansión ósea y a menudo está relacionado con dientes retenidos, con mayor frecuencia caninos o incisivos laterales ${ }^{4}$. El objetivo del artículo fue describir las características clínicas del tumor odontogénico adenomatoide y contrastarlo con los casos descritos en la literatura. Al presentarse este tumor en una paciente pediátrica, no coincide con lo descrito en la literatura debido al rango de edad.

\section{Reporte del caso}

Una paciente femenina de 8 años, acude acompañada de su madre, al servicio de Cirugía Maxilofacial del Hospital Regional Honorio Delgado de Arequipa, quien refiere que su hija presenta un aumento de volumen en la región derecha de su cara (Figura 1); con un tiempo de evolución de la enfermedad de 3 meses aproximadamente, de crecimiento lento, progresivo y asintomático. La madre no refiere ningún antecedente de importancia. A la evaluación extraoral, presentaba una tumoración en la región geniana derecha, con ligero desvanecimiento del surco nasogeniano ipsilateral; no había cambios de coloración ni presentaba linfadenopatias regionales (Figura 1). En la evaluación intraoral, se apreciaba una tumoración que se extendía desde el incisivo lateral derecho hasta el primer molar derecho, sin cambio de coloración, de consistencia indurada, no dolorosa a la palpación y las piezas dentarias no presentaban movilidad (Figura 2).

En la radiografía panorámica (Figura 3) se aprecia una imagen radiolúcida localizada en el maxilar superior del lado derecho que se extiende desde distal de la pieza 12 hasta distal de la pieza 16 y en sentido cefálico cercano al reborde infraorbitario y hacia caudal hasta el reborde alveolar; se encuentra bien delimitado, de borde corticalizados y asociado al canino superior derecho. En la tomografía Cone Beam se aprecia una imagen isodensa con desplazamiento y adelgazamiento de la pared anterior y pared lateral del seno maxilar derecho; se encuentra bien corticalizada y bien delimitada, desplaza el piso del seno maxilar derecho (Figura 4). El diagnóstico diferencial incluye al tumor odontogénico adenomatoide, quiste dentígero y ameloblastoma uniquístico. Se realizó la extirpación quirúrgica bajo anestesia general, se diseńó un abordaje triangular con una descarga hacia mesial. Una vez ubicado el tumor se procedió a realizar la osteotomía y a través de ella se retiró la lesión (Figura 5). El tumor presentaba en su interior el órgano dentario de la pieza 13; no había compromiso con el seno maxilar (Figura 6). Se suturo con vycril 3/0. El examen histológico fue positivo para tumor odontogénico adenomatoide. Se realizó controles periódicos hasta 3 meses después de su intervención quirúrgica no evidenciándose recidiva (Figuras 7 y 8 ).

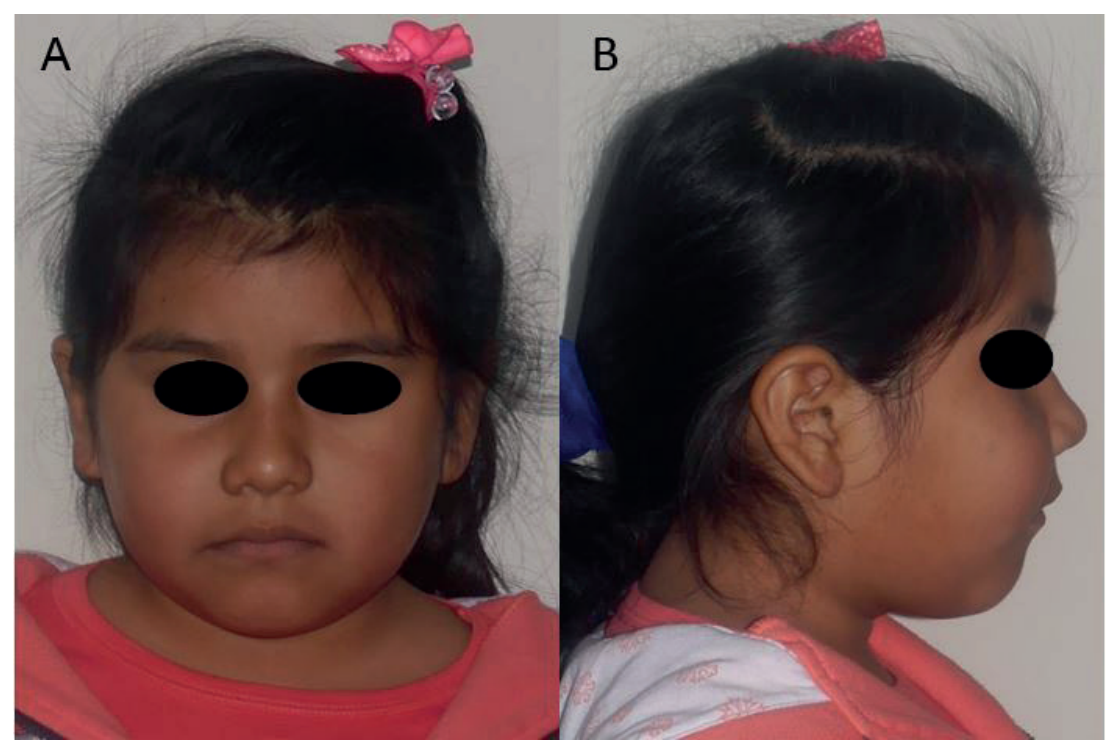

Figura 1. Fotografías extraorales. A) Frontal. B) Lateral 


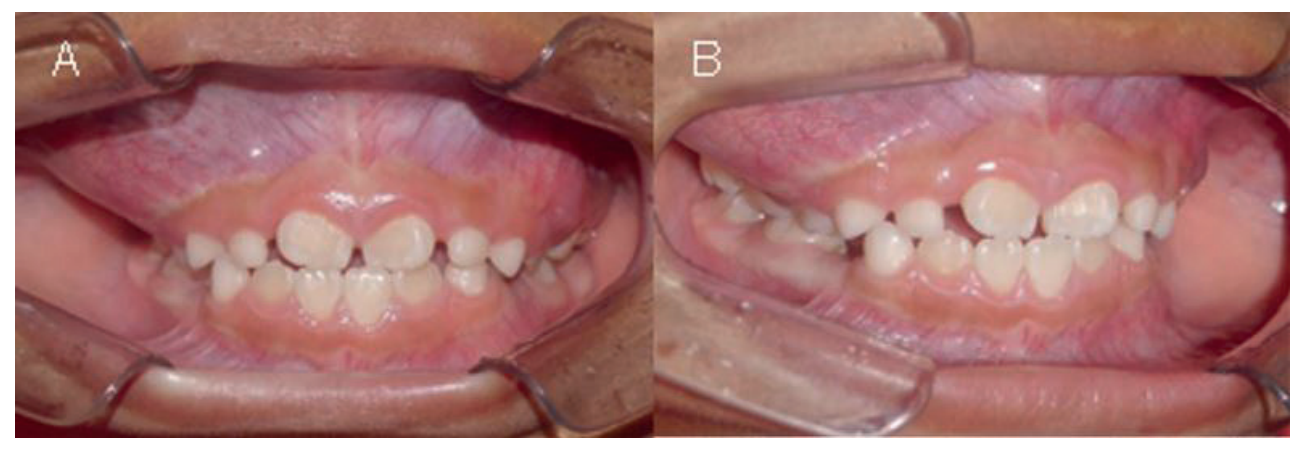

Figura 2. Fotografías intraorales. A) Frontal. B) Lateral

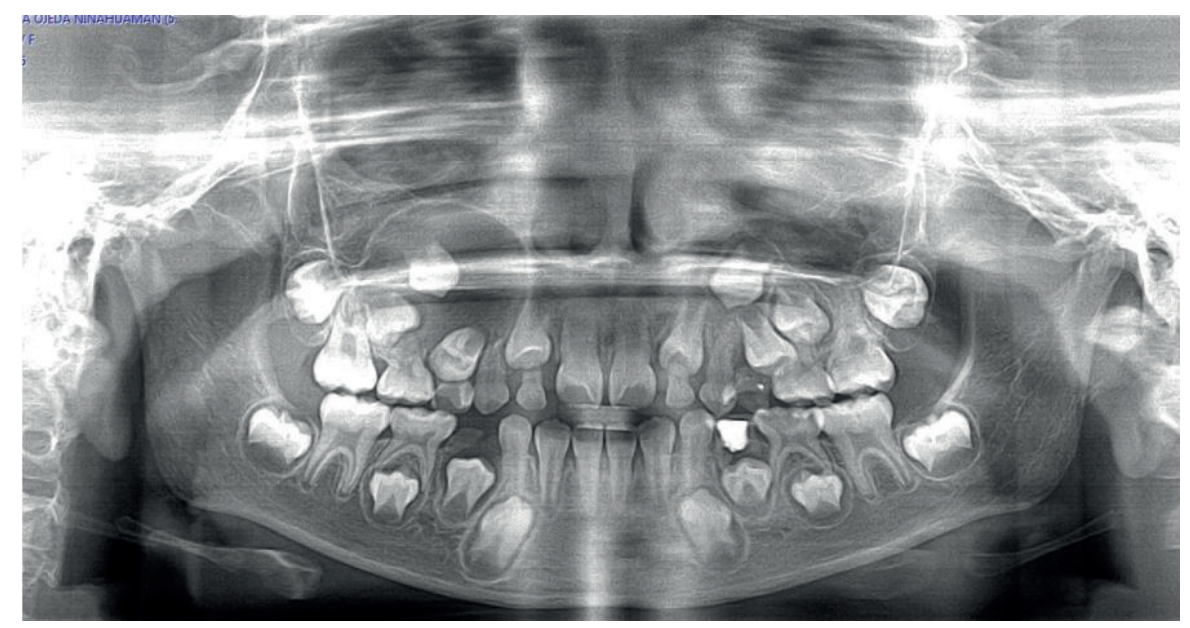

Figura 3. En la radiografía panorámica se observa una imagen radiolúcida localizada en el maxilar superior lado derecho que se extiende desde distal de la pieza 12 hasta distal de la pieza 16

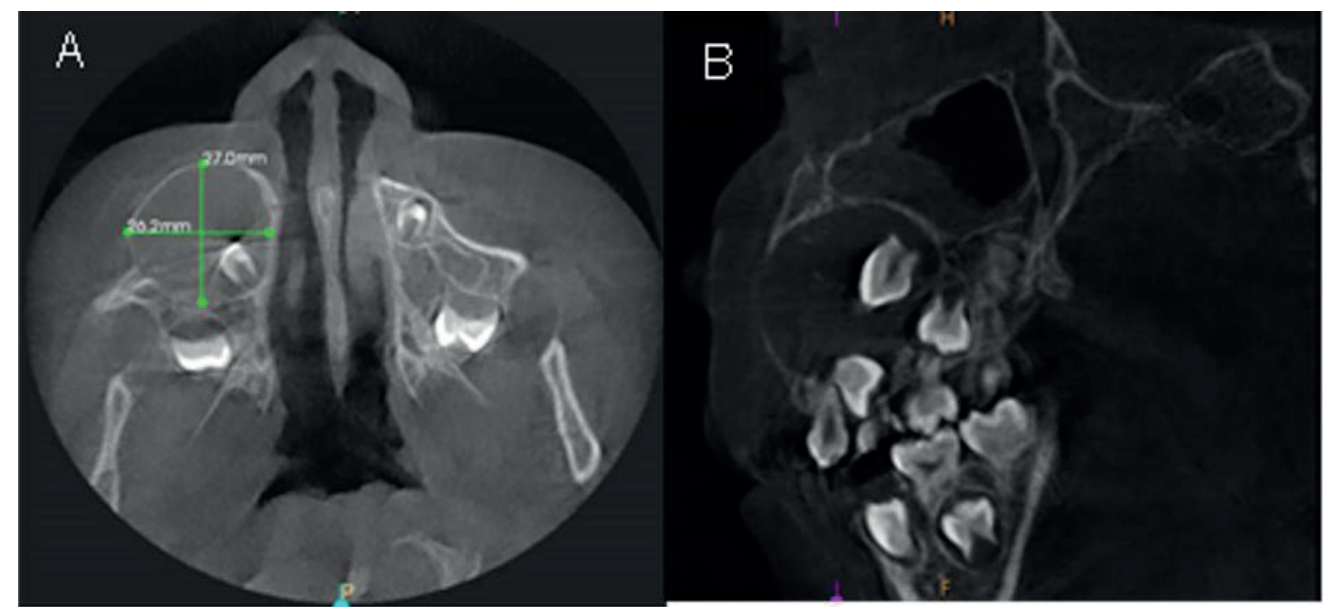

Figura 4. A) En el corte axial de la TCCB, la imagen isodensa tiene un diámetro de $27 \mathrm{~mm}$ y en su interior se observa una pieza dentaria. B) En el corte sagital de la TCCB, la mucosa del seno maxilar se encuentra desplazada hacia caudal 


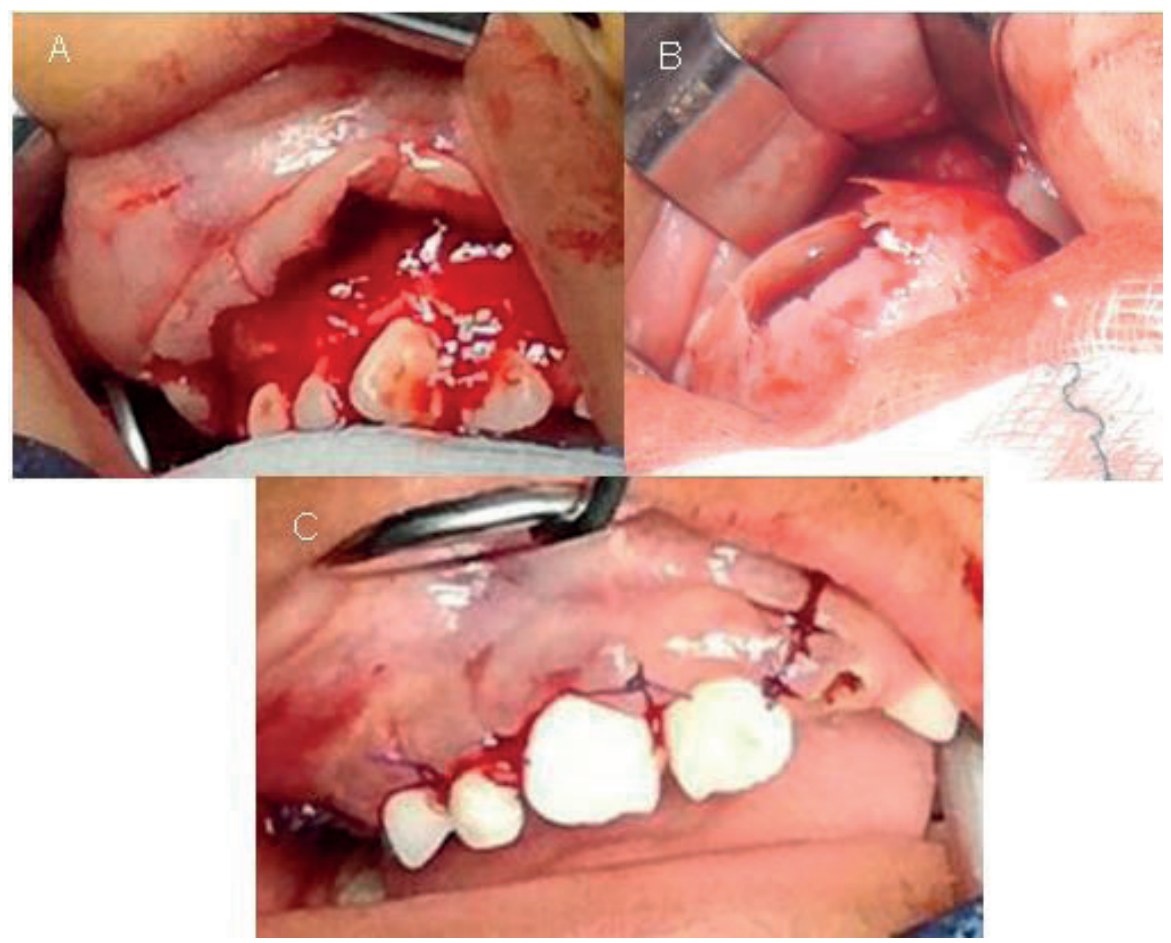

Figura 5. A) Se realiza un abordaje triangular. B) Levantamiento del colgajo. C) El cierre del abordaje, sin tensión

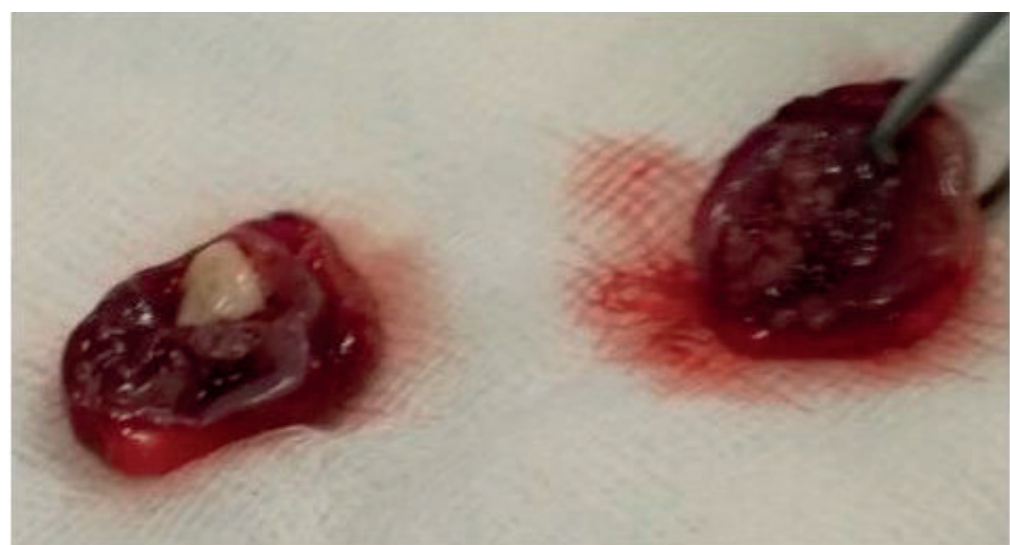

Figura 6. En el interior de la tumoración, se encontraba una pieza dental retenida

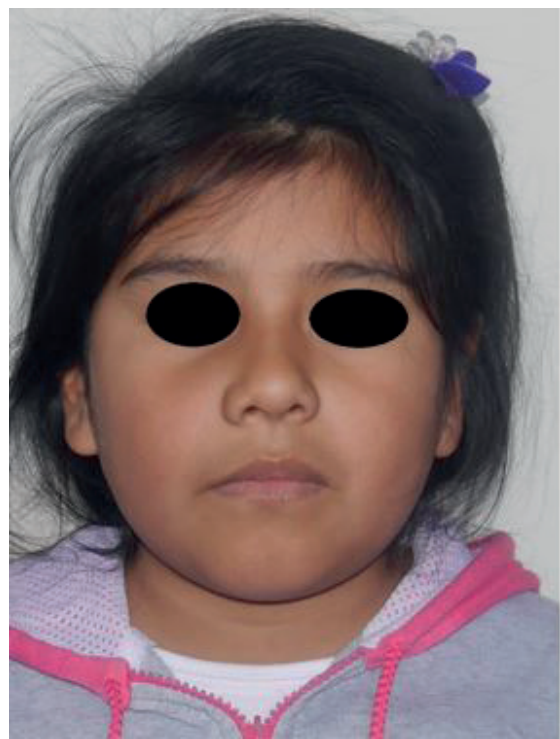

Figura 7. Fotografía extraoral frontal del control postoperatorio, se observa una remisión de la tumoración en la región nasogeniana derecha 


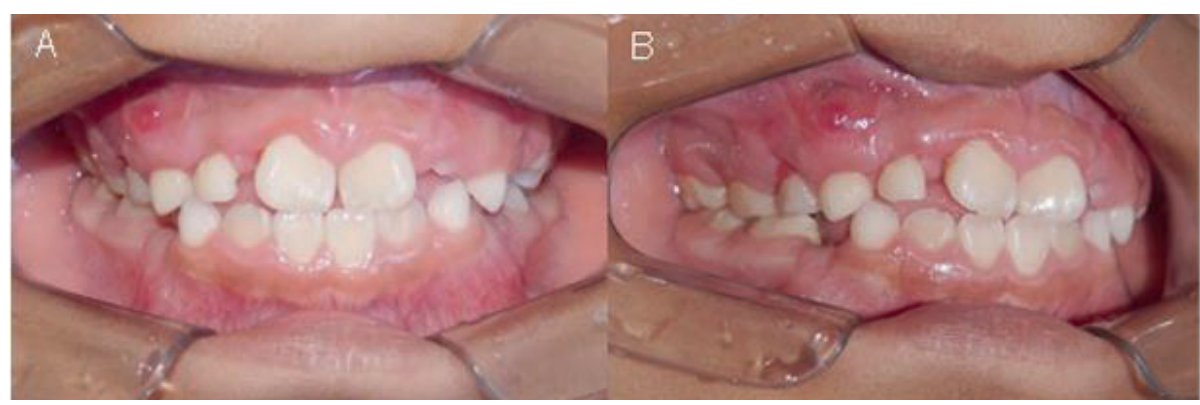

Figura 8. Fotografías intraorales del control postoperatorio. A) Fotografía frontal. B) Fotografía lateral

\section{Discusión}

El TOA es una lesión benigna poco frecuente de origen odontogénico, se presenta principalmente en pacientes jóvenes en la segunda y tercera década de vida; evidenciándose una mayor frecuencia en las mujeres respecto a los varones (2:1), la zona con mayor predilección es el maxilar superior y suele estar asociado a un diente retenido. Algunos estudios han demostrado que este tumor representa sólo el $0,1 \%$ de los tumores y quistes de mandíbula y el 3\% de todos los tumores odontogénicos 5 .

Algunos autores se refieren al TOA como el tumor de los dos tercios debido a que ocurre en el maxilar en aproximadamente $2 / 3$ de los casos, en las mujeres jóvenes se presenta en una relación de $2 / 3$ con respecto a los varones; además $2 / 3$ de los casos son asociados con piezas dentarias no erupcionadas y por último $2 / 3$ de los dientes afectados son los caninos ${ }^{6}$.

Una extensa revisión realizada por Philipsen ${ }^{7}$, quién se basó en 499 casos de diferentes estudios internacionales describe tres variantes de TOA. El tipo folicular es el más común, representa el $70 \%$ aproximadamente de los casos. Implica un diente impactado y a menudo puede confundirse con un quiste dentígero. En la evaluación radiográfica, la variante denominada folicular intraósea del TOA, muestra un entorno radiolúcido unilocular bien delineado asociado a la corona de un diente retenido. La variante extrafolicular no se asocia a un diente retenido. El tipo periférico es raro y a menudo aparece como un fibroma o un épulis en los tejidos gingivales ${ }^{8}$.

El TOA está compuesto por un agrupamiento de nódulos sólidos de células epiteliales, fusiformes o cuboidales; presenta una configuración trabecular o cribiforme por sus espacios parecidos a ductos y bandas de epitelio. La lesión contiene acumulación de material eosinofílico intercelular y cantidades variables de material calcificado. Algunos estudios de inmunohistoquímica realizados en el TOA demuestran que son positivos para AE1/AE3, 34betaE12, CK5, CK14, CK19 '. En concordancia con su benignidad y con su baja tasa de recidiva existe una escasa actividad proliferativa, 2-3\% de núcleos marcados por el antígeno Ki-67, estando la proliferación reducida a pequeños nódulos de células epiteliales (AE1-3+) de núcleos elongados o fusiformes; la mayor incidencia en pacientes de sexo femenino no tiene una causa exacta, se pensaba que era en base a la dependencia de una hormona tumoral; pero ha sido descartado debido a la ausencia de expresión de receptores hormonales (RE y RPg) ${ }^{10}$.
En el diagnóstico diferencial se incluye al quiste dentígero, ameloblastoma uniquístico y el tumor odontogénico epitelial calcificante ${ }^{11}$. Las características radiográficas son de una imagen radiolúcida alrededor de la corona de una pieza dentaria no erupcionada, de bordes definidos y corticalizados. Se pueden presentar áreas opacas (calcificaciones) en la radiografía y puede dar una apariencia de un tumor odontogénico epitelial calcificante ${ }^{12}$; sin embargo, el diagnóstico diferencial de quiste dentígero se excluye en los casos de TOA en los que la radiolucidez se está extendiendo más allá de la unión corona-raíz ${ }^{13}$. Mientras que la imagen radiolúcida unilocular puede aparentar un ameloblastoma uniquístico ${ }^{14}$. En raras ocasiones el tumor odontogénico adenomatoide puede presentarse como imágenes radiolucidas multiloculares, en ese caso el ameloblastoma y el queratoquiste odontogénico pueden considerarse como diagnostico radiológico diferencial ${ }^{15}$.

$\mathrm{Al}$ ser un tumor benigno y presentar una baja tasa de recurrencia, el tratamiento de elección es la enucleación y curetaje, diversos autores describen esta técnica quirúrgica, al considerarlo un manejo conservador de la lesión.

El pronóstico del TOA es bueno, la tasa de recurrencia es de $0,2 \%{ }^{16}$. Además todas las variantes del TOA muestran un comportamiento biológico benigno idéntico y casi siempre se localizan en lesiones bien encapsuladas. Se ha demostrado que la escisión quirúrgica conservadora es la modalidad de tratamiento de elección. En la revisión de la literatura sólo se han reportado cuatro casos de recurrencias.

La evaluación clínica, la localización del tumor, la edad, la evaluación imagenológica es de vital importancia para realizar un buen diagnóstico diferencial.

Se concluye que el reporte de caso que se presentó coincide con lo reportado por Reichart y Philipsen ${ }^{7}$; como las características clínicas, la localización (sector anterior del maxilar superior), la presencia de órganos dentarios retenidos como el canino, el sexo femenino que se presenta con más incidencia; sin embargo con respecto a la edad, en este reporte de caso en particular se presentó a la edad de 8 años, no coincide con lo que se reporta en la literatura, debido a que este tipo de tumor afecta con mayor frecuencia en la segunda y tercera década de vida. El crecimiento del tumor en la paciente fue lento; los exámenes auxiliares también coinciden con lo descrito en la literatura. No presento recidiva después del tratamiento de enucleación y curetaje con una evolución 
favorable. Por lo mencionado es de suma importancia considerar el TOA como diagnóstico diferencial en pacientes pediátricos que presenten un cuadro clínico similar.

\section{Referencias bibliográficas}

1. Philipsen HP, Reichart PA, Zhang H, Nikai Q, Yu QX. Adenomatoid odontogenic tumor: biologic profile based on 499 cases. J Oral Pathol Med 1991;20:149-58.

2. Barnes L, Eveson JW, Reichart P, Sidransky D. World Health Organization Classification of Tumors. Pathology and genetics of head and neck tumors. Lyon: IARC Press; 2005.p.304-5.

3. Philipsen HP, Reichart PA. Adenomatoid odontogenic tumor: facts and figures. Oral Oncol. 1999;35:125-31. DOI: $10.1016 / S 1368-8375(98) 00111-0$

4. M.K. Jawanda, K.R. Anand, N. Mathur, P. Gupta, S. Jain. Extrafollicular adenomatoid odontogenic tumour presenting as a periapical lesion: report of a rare entity, Int. J. Pediatr. Otorrinolaringol. 2014;9(2):47-51. DOI: 10.1016/j.pedex.2014.01.001

5. Lee JK, Lee KB, Hwang BN. Adenomatoid odontogenic tumor: A case report. J Oral Maxillofac Surg. 2000;58(10):1161-4. DOI: 10.1053/joms.2000.9581

6. Reichart P, Philipsen H. Odontogenic Tumors and Allied Lesions. London: Quintessence; 2004.

7. Philipsen HP, Reichart PA, Zhang KH, Nikai H, Yu QX. Adenomatoid odontogenic tumor: biological profile based on 499 cases. J Oral Pathol Med. 1991;20(4):14958. DOI: 10.1111/j.1600-0714.1991.tb00912.x

8. Batra P, Prasad S, Parkash H. Adenomatoid odontogenic tumour: review and case report. J Can Dent Assoc. 2005;71:250-3.

9. Nascimento AB, Nonaka C, Weege F. Immunoexpression of BMP-2 and BMP-4 and their receptors, BMPR-IA and BMPR-II, in ameloblastomas and adenomatoid odontogenic tumors. Arch Oral Biol 2017;73:223-229. DOI: $10.1016 /$ j.archoralbio.2016.10.022.
10. Vera-Sempere FJ, Artes-Martínez MJ, Vera-Sirera B, Bonet-Marco J. Follicular adenomatoid odontogenic tumor: Immunohistochemical study. Med Oral Patol Oral Cir Bucal. 2006;11:E305-8.

11. Belgaumi UI, Parkar MI, Malik NA, Suresh KV, Havewala AM, Bhalinge PM. Follicular adenomatoid odontogenic tumor in mandible: a rare case report. Ann Med Health Sci Res. 2015;5:469-472. DOI: 10.4103/21419248.177976

12. Rosa AC, Soares AB, Furuse C, Lima SR, de Araujo VC, Passador-Santos F. A combined epithelial odontogenic tumor? A 7-year follow-up case. Head Neck Pathol. 2017;11(4):519-24. DOI: 10.1007/s12105-016-07679

13. Becker T, Buchner A, Kaffe I. Critical evaluation of the radiological and clinical features of adenomatoid odontogenic tumour. Dentomaxillofac Radiol. 2012;41:533540. DOI: $10.1259 / \mathrm{dmfr} / 19253953$

14. Narayanan VS, Naidu G, Ragavendra R, Mhaske-Jedhe S, Haldar M. Adenomatoid odontogenic tumor of the mandible with unusual radiographic features: a case report. Imaging Sci Dent. 2013;43:111-5. DOI: 10.5624/ isd.2013.43.2.111

15. Nascimento $A B$, Nonaka $C$, Weege F. Immunoexpression of BMP-2 and BMP-4 and their receptors, BMPR-IA and BMPR-II, in ameloblastomas and adenomatoid odontogenic tumors. Arch Oral Biol. 2017;73:223-229. DOI: $10.1016 /$ j.archoralbio.2016.10.022

16. Chuan-Xiang Z, Yan G. Adenomatoid odontogenic tumor: a report of a rare case with recurrence. J Oral Pathol Med. 2007;36:440-3. DOI: 10.1111/j.16000714.2007.00521.x 